Nota de investigación

\title{
La conductividad eléctrica de la solución nutritiva modifica rendimiento y calidad de frutos de Physalis peruviana
}

\author{
Paola Magdalena Colli-Cortés ${ }^{1}$ \\ Manuel Sandoval-Villa ${ }^{1 \S}$ \\ Nieves Rodríguez-Mendoza ${ }^{1}$ \\ Diana Guerra-Ramírez ${ }^{2}$
}

${ }^{1}$ Colegio de Postgraduados-Programa de Edafología-Campus Montecillo. Carretera Mex-Tex km 36.5, Texcoco, Estado de México. CP. 56230. (colli.paola@colpos.mx; marinie@ colpos.mx). ${ }^{2}$ Universidad Autónoma Chapingo-Programa Doctoral en Ciencias Agroalimentarias. Carretera Mex-Tex km 38.5, Chapingo, Texcoco, México. CP. 56230. (dg_bonita33@yahoo.com.mx).

${ }^{\S}$ Autor para correspondencia: msandoval@colpos.mx.

\section{Resumen}

La uchuva (Physalis peruviana L.) es una solanácea susceptible a enfermedades del suelo, por esa razón se propone su cultivo en México bajo invernadero e hidroponía. Es necesario, determinar su respuesta a la conductividad eléctrica de la solución nutritiva $(\mathrm{CE})$, razón por la cual se expuso a las plantas a 1, 2 y $3 \mathrm{dS} \mathrm{m}^{-1}$. La luz solar es otro factor que incide en la calidad de los frutos, por lo cual se cosecharon frutos del interior y exterior del dosel vegetal. También se determinó el efecto de la madurez del fruto. A los frutos se les determinó peso, Brix, firmeza y color (L, a y b). La CE, ubicación en dosel y madurez de frutos generaron 12 tratamientos. Los datos se compararon con prueba de medias de rangos. De los frutos, la CE de la solución nutritiva afectó la firmeza y los Brix excepto el peso. La posición de los frutos en el dosel vegetal tampoco afectó estas variables. La madurez a la cosecha afectó la calidad de los frutos. Los frutos cosechados 30 días después de madurez comercial perdieron peso y firmeza, y aumentaron Brix en frutos más maduros. La ubicación del fruto y madurez no afectaron el color en fruto.

Palabras clave: dosel, madurez, uchuva.

Recibido: abril de 2020

Aceptado: mayo de 2020 
Durante el desarrollo de los frutos ocurren procesos metabólicos que son determinantes en su calidad, estos procesos dependen de factores que no siempre son favorables; por ejemplo, la disponibilidad de nutrientes, la temperatura, incidencia solar, sanidad, entre otros. Los frutos se cosechan y comercializan buscado mantener una calidad óptima durante su vida de anaquel y disminuir los efectos indeseables durante su vida poscosecha.

La etapa de madurez en que son cosechados es un factor determinante en la calidad de los frutos de uchuva ya que esta determina el sabor y textura. La conductividad eléctrica (CE) de la solución nutritiva es determinante en la calidad de los frutos, porque está relacionada con la fertilidad del suelo y la salinidad. En algunos estudios se ha encontrado que si la CE es alta puede haber efectos negativos para los cultivos porque se afecta la asimilación de $\mathrm{CO}_{2}$ y transpiración, ya que a alta $\mathrm{CE}$ ocurre la absorción excesiva de $\mathrm{Na}^{+} \mathrm{y} \mathrm{Cl}^{-}$y ocurre desequilibrio en la disponibilidad de nutrientes (Wu y Kubota, 2008).

\section{Diseño experimental}

El experimento se realizó en un invernadero en el Colegio de Postgraduados. Se diseñó un experimento con tres tratamientos de conductividad eléctrica (CE) de la solución Steiner: 1, 2 y 3 $\mathrm{dS} \mathrm{m}{ }^{-1}$. Cada tratamiento constó de nueve repeticiones con un total de 27 plantas en un diseño completamente al azar. Asimismo, se consideraron ubicación del fruto en el dosel vegetal (interna y externa) y dos fechas de cosecha de fruto: a madurez comercial (MC) y 30 días después de MC (MC30).

\section{Cosecha}

Se seleccionaron y etiquetaron frutos de la parte interna (I) como en la parte externa (E) del dosel de la planta en cada tratamiento. Se cosecharon frutos cuando alcanzaron el estado de madurez comercial (MC) o estado 6 según la norma Técnica Colombiana 4580 (NTC) (ICONTEC, 1999). Treinta días después del estado 6 se cosecharon frutos (MC30) del interior del dosel vegetal y del exterior de este. Los frutos se pesaron, se determinaron grados Brix con un refractómetro, color con un colorímetro de reflexión Hunter Lab, y textura con un texturómetro Wagner Force Five modelo FDV-30.

\section{Análisis estadístico}

El proceso de los datos inició con un análisis no paramétrico por medio de la prueba de Kruskal Wallis. El modelo estadístico consideró los factores; 1) conductividad eléctrica de solución Steiner (1, 2 y $\left.3 \mathrm{dS} \mathrm{m}^{-1}\right)$; 2) posición del fruto en el dosel la planta (internos y externos); y 3) grado de madurez del fruto (madurez comercial y madurez comercial más 30 días). Se realizó una comparación de medias para elegir el mejor tratamiento. Se utilizó el programa Statistical Analysis System (SAS versión 9, 2002).

\section{Peso de frutos}

El peso promedio de los frutos fue de $3 \mathrm{~g}$. Los frutos con mayor peso fueron los que se encontraban en madurez comercial ( $p<0.05$ ): de 2.7 a $3 \mathrm{~g}$, los frutos en MC30 tuvieron pesos entre 2.4 y $2.9 \mathrm{~g}$ (Figura 1 y 2). 


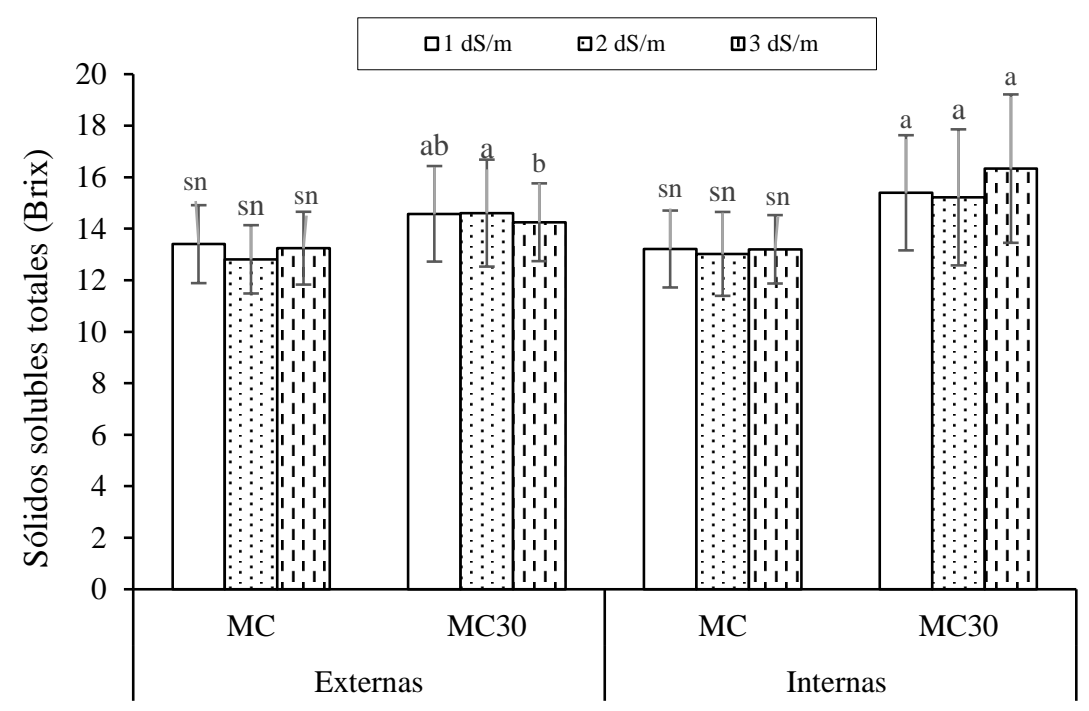

Figura 1. Sólidos solubles totales (Brix) en frutos de uchuva por efecto de conductividad eléctrica de la solución nutritiva, posición en el dosel vegetal (externos e internos) y madurez del fruto $(\mathbf{M C}=$ madurez comercial, $\mathrm{MC30}=30$ días después de $\mathrm{MC})$. Medias con la misma letra, indican que las diferencias no son significativas.

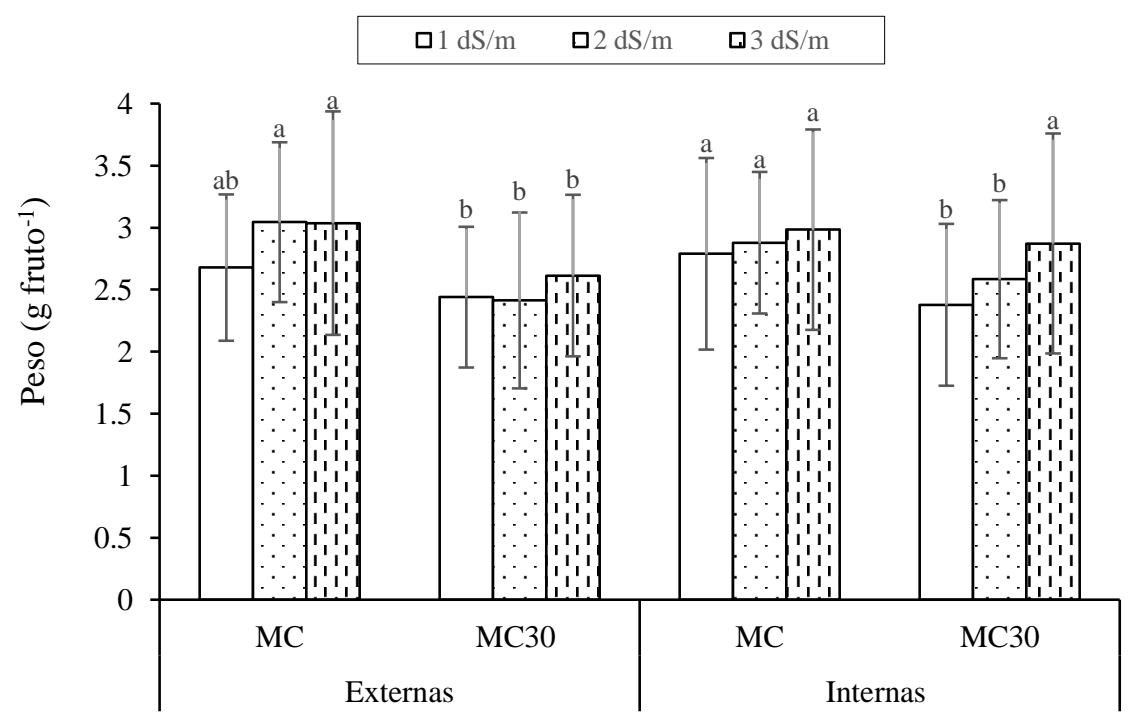

Figura 2. Peso de los frutos de uchuva por efecto de conductividad eléctrica de la solución nutritiva, posición en el dosel vegetal (externos e internos) y madurez del fruto $(\mathrm{MC}=$ madurez comercial, $\mathbf{M C 3 0}=\mathbf{3 0}$ días después de $\mathbf{M C}$ ). Medias con la misma letra, indican que las diferencias no son significativas.

La pérdida de peso en los frutos MC30 se atribuye a compuestos que favorecen la debilitación de la pared celular: pectinmetilesterasas y $\alpha$-galactosidasas, las cuales, según Trinchero et al. (1999), aumentan su concentración cuando los frutos de uchuva están madurando. No hubo efecto de la posición del fruto en el dosel. Fischer et al. (2014) indican que una buena incidencia solar proporciona condiciones para obtener frutos de buena calidad. 
Muniz et al. (2014) afirman que los frutos de Physalis necesitan de 1500 a 2000 h de luz al año para obtener una buena calidad en los frutos. Frutos de CE-3 y MC30 fueron los únicos que mostraron diferencias de peso, por efecto de posición, donde los frutos externos tuvieron pesos de $2.6 \mathrm{~g}$ y los internos $2.9 \mathrm{~g}$, contrario a lo que se esperaba. Esto pudo deberse a que en la parte interna cálices y hojas cercanas transpiran menos y se ahorran fotoasimilados que son traslocados a los frutos cercanos (Mazorra, 2003).

En los tratamientos MC-E, MC30-E y MC-I la CE no afectó el peso de los frutos (Figura 3); sin embargo, los frutos MC30-I mostraron mayor peso con CE-3 que con CE-1 y CE-2 con la misma madurez y posición del fruto dentro del dosel vegetal. Aguilar-Carpio et al. (2018) obtuvieron resultados similares: frutos con concentración de 150\% de la solución Steiner tuvieron 59\% más peso respecto a frutos con $50 \%$ de la solución Steiner.

Los tratamientos con alta CE tienen elevada productividad de papa $\left(2.2 \mathrm{dS} \mathrm{m}^{-1}\right.$ ) (Calori et al., 2016). Por otra parte, Lee et al. (2015) mencionan que alta CE disminuye la absorción de agua y afecta la tasa respiratoria de las plantas. Los frutos en MC y MC30 fueron de $2.9 \mathrm{~g}$ y $2.6 \mathrm{~g}$, esto afirma que los frutos en MC tienen mayor peso $(p<0.05)$. El peso promedio de los frutos externos e internos fue de $2.7 \mathrm{~g}$ y sin efecto de posición en dosel ( $p>0.05)$. Álvarez-Herrera et al. (2015) obtuvieron frutos con $3.33 \mathrm{~g}$.

\section{Grados Brix}

En frutos MC30 se encontraron altos valores de Brix (Figura 3), no hubo efecto de la posición del fruto dentro del dosel vegetal. Tampoco hubo diferencias significativas en Brix por CE $(p>0.05)$. Los frutos MC variaron entre $12.8 \pm 1.4$ y $13.4 \pm 1.6$ con promedio de $13.1 \pm 1.5$ Brix. Según la NTC 4580, los frutos en madurez comercial deben tener 15.1 Brix, este dato es cercano a lo que se obtuvo en frutos cosechados MC30, con Brix entre $14.3 \pm 1.5$ y $16.3 \pm 2.9$ y promedio de $15.1 \pm 2.4$.

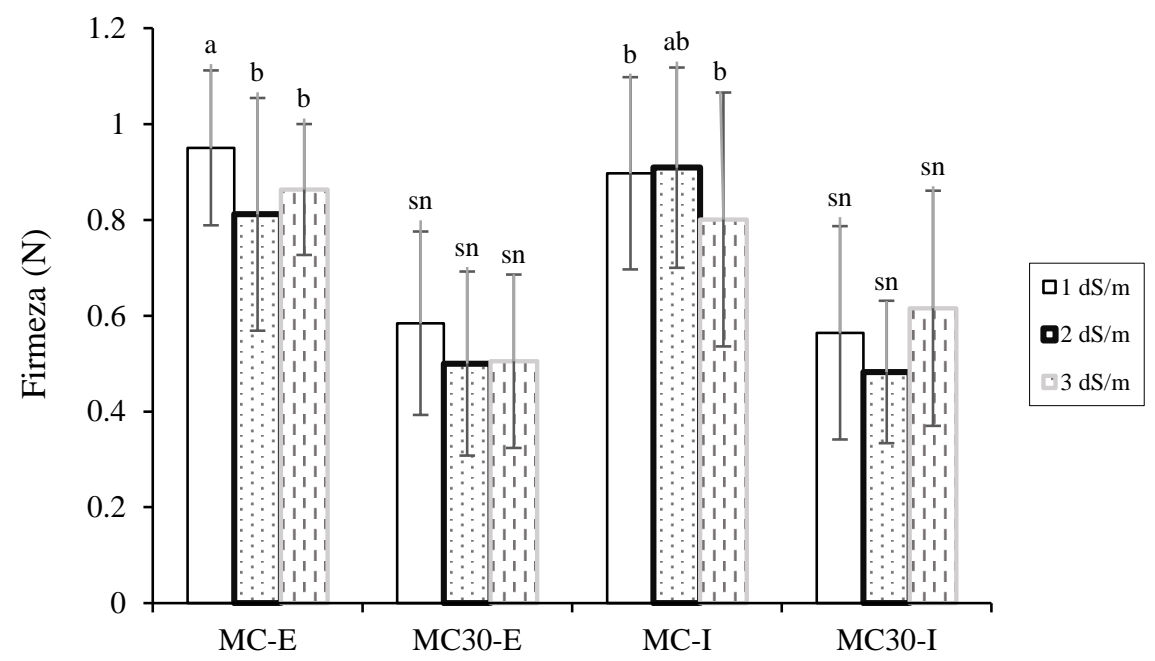

Figura 3. Firmeza de frutos de uchuva por efecto de la conductividad eléctrica de la solución nutritiva, posición en el dosel vegetal (externos e internos) y estado de madurez (MC $=$ madurez comercial, MC30 = 30 días después de MC). Medias con la misma letra, indican que las diferencias no son significativas. 
Se encontraron valores similares en frutos de uchuva ecotipo Colombia provenientes de cultivos para exportación por Pinzón et al. (2015) donde obtuvieron valores de 14.5 y 15.8 Brix almacenados por 18 días a 2 y $4 \mathrm{C}$ respectivamente. Lo cual podría indicar que los Brix obtenidos en los frutos MC30 son similares, con el mismo efecto en frutos almacenados a bajas temperaturas. Bravo et al. (2015) encontraron valores similares en frutos en MC (S3-S5 según NTC4589) con 14.17 y 13.3 Brix.

Pinzón et al. (2015) registró 17.3 Brix en frutos almacenados a temperatura ambiente por 15 días que es cercano a lo obtenido en el tratamiento 3-MC30-I (16.3 Brix). Estas similitudes pueden ser atribuidas a que en ambos casos se generaron procesos metabólicos naturales donde aumentan azúcares como la sacarosa, glucosa y fructosa (Duque et al., 2011), en el primer caso por no someterse a algún tratamiento poscosecha y en el segundo a que había mayor sustrato para generar azúcares ya que se encontraban en la parte interna y con mayor $\mathrm{CE}$.

Singh (2012) relaciona los sólidos solubles totales con el incremento de sustancias pépticas totales cuando los frutos están en proceso de madurez (1 a 8 semanas después de la antesis). Por otra parte, Balaguera-López et al. (2015) mencionan que el cáliz de los frutos puede ser una fuente importante de carbohidratos, a lo que se podría deber la transformación de estos carbohidratos en azúcares. Los frutos, externos e internos, tuvieron de 13.77 a 14.4 Brix, por lo que no hubo efecto por la posición en Brix de los frutos.

\section{Firmeza de los frutos}

La firmeza en frutos en MC contra MC30 indica que los últimos disminuyeron su firmeza (Figura 4). Singh (2012) menciona que durante los primeros estadios de desarrollo (de una a ocho semanas después de la antesis) los frutos aumentan su firmeza de forma constante. Majumder (2002) menciona que hay un incremento de sustancias pécticas durante el desarrollo de los frutos y estas son responsables de formar nuevas células en la pared celular. Majumder (2002) encontró que esto se debe a la solubilización de las sustancias pécticas, por la síntesis de enzimas como la poligalacturonasas (PG), que a su vez están relacionadas con la presencia de etileno.

La firmeza en frutos en MC va de $0.8 \pm 0.2$ a $1 \pm 0.2 \mathrm{~N}$, para frutos en MC30 se obtuvieron de 0.5 \pm 0.2 a $0.7 \pm 0.7 \mathrm{~N}$. La firmeza en los frutos en MC, MC30, Externos e Internos tuvieron de 0.87a, $0.57 \mathrm{~b}, 0.73$ c y $0.71 \mathrm{c} \mathrm{N}$, respectivamente, sin considerar la CE. Estos valores están por debajo de lo encontrado por Pinzón et al. (2015), 3.4 y 2.3 N bajo dos diferentes temperaturas con 15 días de almacenamiento.

El tratamiento de mayor firmeza en frutos fue el MC-E con CE de $1 \mathrm{dS} \mathrm{m}^{-1}$ (Figura 4), con una firmeza de $1 \mathrm{~N}$, en los demás frutos en MC no hubo efecto. Los frutos con CE-2 MC30-I y CE3 MC30-E fueron los de mayor pérdida de firmeza, $0.5 \mathrm{~N}$. Aunque en frutos MC30 no hubo influencia de la CE ( $p>0.05$ ), el tratamiento que conservó su firmeza fue el CE-2 MC30-E. Los frutos externos tuvieron mayor firmeza en CE-1 MC. Los frutos MC30-E tuvieron su mayor firmeza en CE-2 y CE-1.

Los frutos MC30 pierden su firmeza (Figura 4a). Balaguera-López et al. (2015) encontraron una tendencia similar con frutos con y sin cáliz donde la firmeza se pierde cuando se almacenaron por 15 días a temperatura ambiente. La firmeza en MC30 no fue afectada por la CE (Figura 4b). 

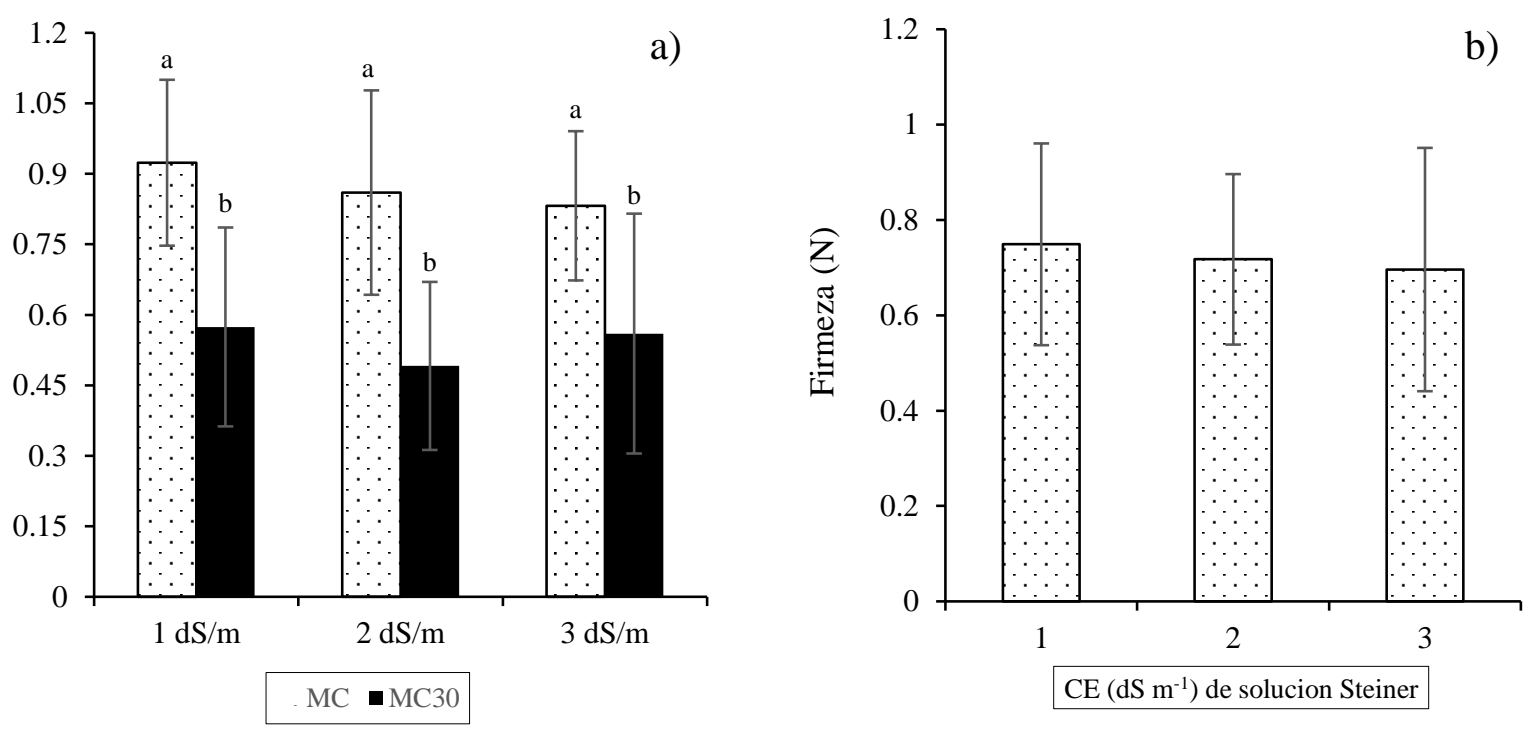

Figura 4. Efecto de la conductividad eléctrica (CE) en la firmeza de frutos de uchuva por efecto de la fecha de cosecha de los frutos (a); y conductividad eléctrica de la solución nutritiva (b). Medias con la misma letra, indican que las diferencias no son significativas.

\section{Color de los frutos}

Respecto a L los tratamientos CE-2-MC30-I, CE3-MC30-I, CE1-MC-I, CE1-MC-E fueron los de mayor luminosidad. Estos frutos se encontraban en la parte interna del dosel vegetal. No se observó tendencia respecto a la CE y madurez de los frutos. La característica a del color no fue afectada por CE y madurez de frutos $(p>0.05)$. La característica b tuvo mayores valores en los tratamientos CE1-MC-I, Ce3-MC30-I, CE1-MC-E y Ce2-MC30-I, al igual que para L, no se encontraron tendencias por $\mathrm{CE}$, ni para madurez de los frutos, como refiere el Cuadro 1.

Cuadro 1. Características de color de los frutos por efecto de la madurez de cosecha (MC madurez comercial y 30 días después de MC; MC30), posición de los frutos en el dosel y por conductividad eléctrica (CE) en la solución nutritiva.

\begin{tabular}{|c|c|c|c|c|c|c|c|}
\hline \multirow{2}{*}{$\begin{array}{c}\mathrm{CE} \\
\left(\mathrm{dS} \mathrm{m} \mathrm{m}^{-1}\right)\end{array}$} & Posición & \multicolumn{3}{|c|}{ Externa al dosel } & \multicolumn{3}{|c|}{ Interna al dosel } \\
\hline & Madurez & $\mathrm{L}$ & $\mathrm{a}$ & $\mathrm{b}$ & $\mathrm{L}$ & $\mathrm{a}$ & $\mathrm{b}$ \\
\hline \multirow[t]{2}{*}{1} & $\mathrm{MC}$ & $59 \pm 4.6 \mathrm{a}$ & $19 \pm 5.6$ & $50.6 \pm 9.7 \mathrm{a}$ & $59.2 \pm 2.5 \mathrm{a}$ & $21.4 \pm 2.6$ & $54.3 \pm 2.7 \mathrm{a}$ \\
\hline & MC30 & $53.3 \pm 3.4 \mathrm{c}$ & $20 \pm 5.5$ & $46.8 \pm 11 \mathrm{~b}$ & $51.8 \pm 4.3 \mathrm{c}$ & $20.2 \pm 3.8$ & $46.9 \pm 7.5 \mathrm{~b}$ \\
\hline \multirow[t]{2}{*}{2} & MC & $54.9 \pm 4.2 \mathrm{~b}$ & $19 \pm 6.1$ & $42.5 \pm 11 \mathrm{~b}$ & $53.5 \pm 4 c$ & $19.8 \pm 4.3$ & $44.7 \pm 8.5 b$ \\
\hline & MC30 & $56.3 \pm 5.6 \mathrm{~b}$ & $16.1 \pm 5.5$ & $42.5 \pm 13 \mathrm{~b}$ & $60.3 \pm 4 \mathrm{a}$ & $20.3 \pm 4.4$ & $50.4 \pm 8.3 \mathrm{a}$ \\
\hline \multirow[t]{2}{*}{3} & $\mathrm{MC}$ & $55.8 \pm 4.5 \mathrm{~b}$ & $19 \pm 5$ & $43.4 \pm 1 b$ & $53.5 \pm 5 \mathrm{~b}$ & $22.2 \pm 2.7$ & $49.5 \pm 4 b$ \\
\hline & MC 30 & $58.4 \pm 3.2 \mathrm{a}$ & $18.3 \pm 6.4$ & $48.5 \pm 12 b$ & $59.5 \pm 3.5 \mathrm{a}$ & $20.8 \pm 3$ & $53.6 \pm 2.7 \mathrm{a}$ \\
\hline
\end{tabular}




\section{Conclusiones}

La conductividad eléctrica (CE) de la solución nutritiva no afecta el peso y los Brix en frutos de uchuva. La CE afecta la firmeza de los frutos: es mayor con CE de $1 \mathrm{dS} \mathrm{m}^{-1}$ con frutos cosechados en madurez comercial y del exterior del dosel. La posición de los frutos en el dosel vegetal (externa e interna) no afecta en el peso, Brix y firmeza de los frutos.

Conforme los frutos están más maduros disminuye peso y firmeza, y aumentan los Brix. La CE de la solución nutritiva no afecta en el color de los frutos, pero la posición y madurez inducen diferencias para L (luminosidad) y b (tono desde amarillo a azul) excepto en a (tono de verde a amarillo).

\section{Literatura citada}

Aguilar Carpio, C.; Juárez López, P.; Campos Aguilar, I.; Alia Tejacal, I.; Sandoval Villa, M. y López-Martínez, V. 2018. Análisis de crecimiento y rendimiento de uchuva (Physalis peruviana L.) cultivada en hidroponía e invernadero. Rev. Chapingo Ser. Hortic. 24(3):83-94. http://dx.doi.org/10.5154/r.rchsh.2017.07.024.

Álvarez-Herrera, J. G.; Fischer, G. y Velez, J. 2015. Producción de frutos de uchuva (Physalis peruviana L.) bajo diferentes láminas de riego, frecuencias de riego y dosis de calcio. Rev. Colomb. Cienc. Hortíc. 9(2):222-233. https://doi.org/10.17584/rcch.2015v9i2.4177.

Balaguera-López, H. E.; Martínez, C. A. y Herrera A. A. 2015. Refrigeration affects the postharvest behavior of 1-methylcyclopropenetreated cape gooseberry (Physalis peruviana L.) fruits with the calyx. Agronomía Colombiana. 33(3):356-364. Doi: 10.15446/agron.colomb. v33n3.51896.

Bravo, K.; Sepúlveda-Ortega, S.; Lara-Guzmán, O.; Navas-Arboleda, A. A. y Osorio, E. 2015. Influence of cultivar and ripening time on bioactive compounds and antioxidant properties in Cape gooseberry (Physalis peruviana L.). J. Sci. Food Agric. 95(7):1562-1569. Doi: 10.1002/jsfa.6866.

Calori, A.; Factor, T.; Feltran, J.; Watanabe, E.; Moraes, C. and Purquerio, L. 2016. Electrical conductivity of the nutrient solution and plant density in aeroponic production of seed potato under tropical conditions (winter/spring). Bragantia, Campinas. 76(1):23-32. Doi: 10.1590/1678-4499.022.

Duque, A. L.; Giraldo, G. A. y Quintero V. D. 2011. Caracterización de la fruta, pulpa y concentración de uchuva (Physalis puruviana L.). Temas Agrarios. 16(1):75-83.

Fischer, G.; Almanza-Merchan, P. y Miranda, D. 2014. Importancia y cultivo de la uchuva (Physalis peruviana L.). Rev. Brasileña Frutic. 36(1):1-15. http://dx.doi.org/10.1590/01002945-441/13

ICONTEC. 1999. Instituto Colombiano de Normas Técnicas y Certificación. Frutas frescas. Uchuva. Especificaciones. Norma Técnica Colombiana NTC 4580. Instituto Colombiano de Normas Técnicas y Certificación, Bogotá.

Lee, Y.; Yoo, C.; Park, N. and Yeoung, Y. 2015. Influence of various nutrient concentration on the growth and yield of summer strawberry cultivars cultivated in a hydroponic system. Hortic. Environ. Biotechnol. 56(4):421-426. 
Majumder, K. and Mazumdar, B. 2002. Changes of pectic substances in developing fruits of capegooseberry (Physalis peruviana L.) in relation to the enzyme activity and evolution of ethylene. Sci. Hortic. 96(1):91-101. https://Doi.org/10.1016/S0304-4238(02)00079-1.

Mazorra, M.; Quintana, A.; Miranda, D.; Fischer, G. y Cháves, B. 2003. Análisis sobre el desarrollo y la madurez fisiológica del fruto de la uchuva. Agronomía Colombiana. 21(3):175-189.

Muniz, J.; Kretzchmar, A.; Rufalo, L.; Pelizza, T.; Rufalo, A. and de Macedo, T. 2014. General aspects of physalis cultivation. Ciencia Rural, Santa María. 44(6):964-970. http://dx. Doi.org/10.1590/S0103-84782014005000006.

Pinzón, E.; Reyes, A.; Álvarez-Hernández, J; Leguizamo, M. y Joya, J. 2015. Comportamiento del fruto de uchuva Physalis peruviana L. bajo diferentes temperaturas de almacenamiento. Ver. Cienc. Agríc. 32(2):26-35. doi: http://dx.Doi.org/10.22267/rcia.153202.10.

Singh, D. B.; Pal A. A.; Lal, S.; Ahmed, N. and Mirza, A. 2012. Growth and developmental changes of cape gooseberry (Physalis peruviana L.) fruits. Asian J. Hortic. 7(2):374-378.

Trinchero, G.; Sozzi, G.; Cerri, A.; Vilella, F. and Fraschina, A. 1999. Ripening-related changes in ethylene production, respiration rate and cell-wall enzyme activity in goldenberry (Physalis peruviana L.) a solanaceous species. Posharvest Biol. Technol. 16(2):139-145.

Wu, M. and Kubota, C.2008. Effect of high electrical conductivity of hydroponic nutrient solution and its application timing on lycopene, chlorophyll and sugar concentration of hydroponic tomatoes during ripening. Sci. Hortic. 116(2):122-129. https://doi.org/10.1016/ j.scienta.2007.11.014. 